\title{
Complete genome sequence of a clinical Bordetella pertussis isolate from Brazil
}

\author{
Bruno Gabriel N Andrade', Michel F Abanto Marin'1, Diego Duque Cambuy', \\ Erica Lourenço Fonseca ${ }^{1 /+}$, Nadjla Ferreira Souza ${ }^{2}$, Ana Carolina P Vicente ${ }^{1}$
}

Laboratório de Genética Molecular de Microorganismos, Instituto Oswaldo Cruz-Fiocruz, Rio de Janeiro, RJ, Brasil

${ }^{2}$ Laboratório Central de Saúde Pública Dr Milton Sobral, Recife, PE, Brasil

There has been a resurgence in the number of pertussis cases in Brazil and around the world. Here, the genome of a clinical Bordetella pertussis strain (Bz181) that was recently isolated in Brazil is reported. Analysis of the virulence-associated genes defining the pre-and post-vaccination lineages revealed the presence of the prn2-ptxSIAfim $3 B$-ptxP3 allelic profile in Bz181, which is characteristic of the current pandemic lineage. A putative metallo- $\beta$ lactamase gene presenting all of the conserved zinc-binding motifs that characterise the catalytic site was identified, in addition to a multidrug efflux pump of the RND family that could confer resistance to erythromycin, which is the antibiotic of choice for treating pertussis disease.

Key words: ptxP3 - pandemic lineage - virulence factors - antigenic variant

The genus Bordetella consists of Gram-negative $\beta$-proteobacteria, including the three human pathogens Bordetella pertussis, Bordetella parapertussis and Bordetella bronchiseptica, which are considered the classical Bordetella species (van der Zee et al. 1996). B. pertussis, a fastidious but highly contagious bacterium, is a strict human pathogen and has no known animal or environmental reservoir [for revision, Mattoo and Cherry (2005)].

Whooping cough (pertussis) is a consequence of upper respiratory tract colonisation by $B$. pertussis and is characterised by symptoms such as fever and coughing, which persist for more than a week. During this stage, $B$. pertussis can be isolated and antibiotics are used to control the infection. If the infection persists, a toxaemic stage is achieved, with a characteristic inspiratory gasp (whoop) that is not controlled by antibiotics. Pertussis shows a significant mortality rate in infants and was one of the most frequent and severe disease in this group before the worldwide introduction of an immunisation programme in the $1950 \mathrm{~s}$. Although immunisation with a cellular vaccine was effective in the late XX century, this type of vaccine has been replaced by acellular pertussis vaccines in several countries due to its side effects. In Brazil, pertussis is controlled by the Brazilian National Immunisation Program by administering a whole-cell vaccine; however, acellular vaccines have also been available since 2006. In the 1990s, pertussis reemerged even in countries with highly vaccinated populations and it is currently the most prevalent vaccine-prevent-

doi: 10.1590/0074-0276140288

Financial support: FAPERJ, CNPq, IOC

+ Corresponding author: ericafon@ioc.fiocruz.br

Received 7 August 2014

Accepted 7 October 2014 able disease, representing a public health threat even in developed countries (Hartzell \& Blaylock 2014).

The emergence of strains harbouring allelic variants of the antigens used in vaccine development is one of the possible causes of pertussis resurgence. A number of studies have documented the circulation of strains with allelic variants that differ from those of the vaccine strain (van Loo et al. 2002, Kallonen et al. 2011). In most cases, these alleles present single-nucleotide polymorphisms that result in antigenic divergence relative to the antigens present in the vaccine.

In 2003, the first complete genomes of the classical Bordetella subspecies were published, revealing the genetic determinants of their distinct phenotypes (Parkhill et al. 2003, Bart et al. 2010). A recent study based on whole-genome analyses evaluated $B$. pertussis strains recovered worldwide, though no Brazilian strain was included. This study indicated the existence of at least two major $B$. pertussis lineages related to the pre and post-vaccination periods (Harvill et al. 2013, Bart et al. 2014). Although there has been a lack of characterisation of strains circulating in Brazil, pertussis has also reemerged in this country. Here, we report the first $B$. pertussis genome sequence of a clinical strain isolated in the northeastern region of Brazil (Bz181), in 2013.

The $B$. pertussis Bz181 strain is deposited in the Collection of Bacteria of the Environment and Health at the Oswaldo Cruz Foundation (Fiocruz), Brazil. Whole-genome sequencing of this strain was performed using a Nextera paired-end library on an Illumina HiSeq 2500 sequencer within the high-throughput platform of the Oswaldo Cruz Institute, Fiocruz. The sequencing generated 10,120,624 reads with an average read length of $87 \mathrm{bp}$. The paired-end reads were de novo assembled using the a5 assembly pipeline, which yielded a database composed of 295 contigs, totalling $\sim 3.82 \mathrm{Mb}$, with an average $\mathrm{G}+\mathrm{C}$ content of $67.7 \%$. Gene prediction and annotation were performed with the NCBI Prokaryotic Genome Automatic Annotation Pipeline. An overall 


\section{Virulence factors classes}

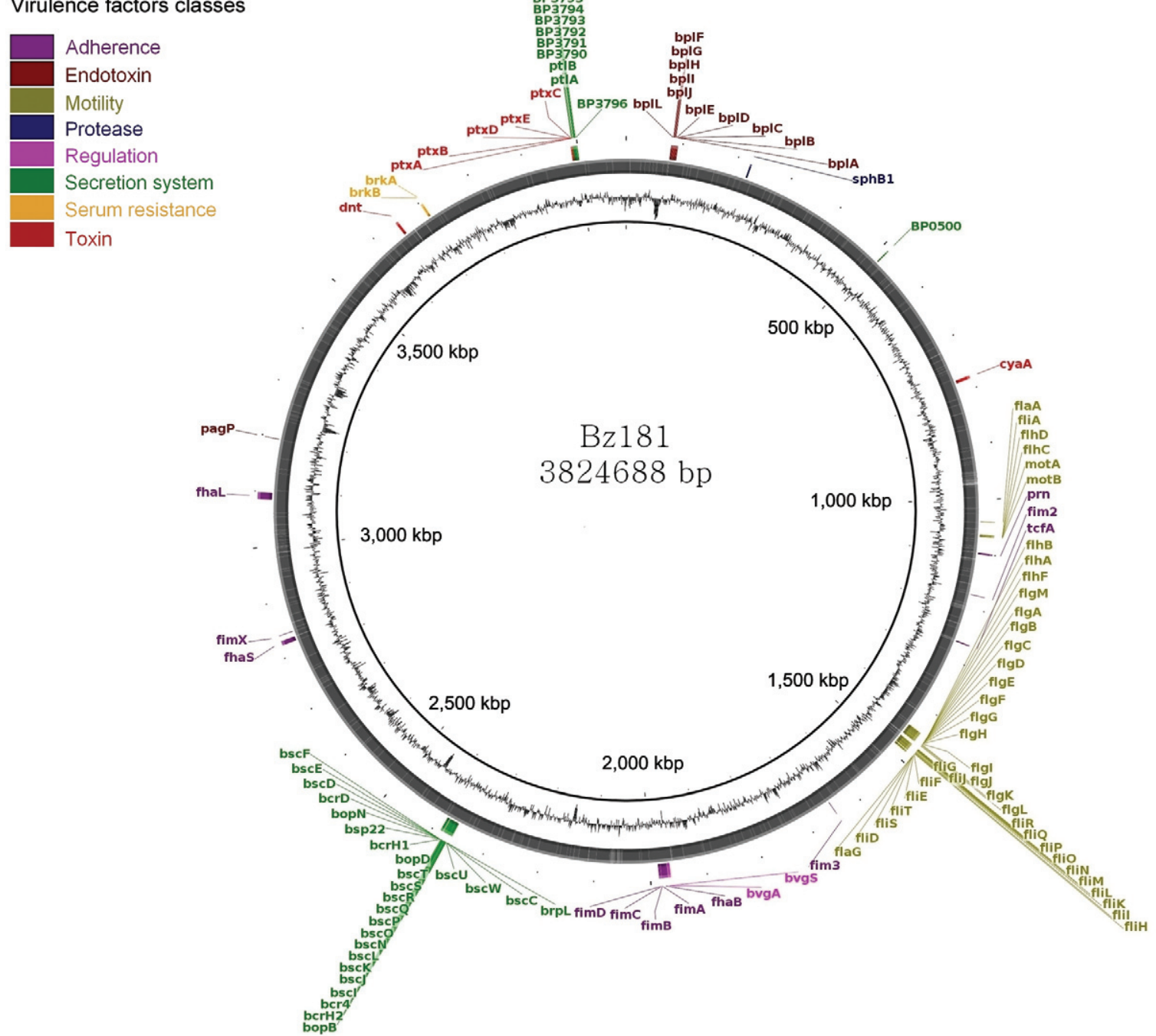

Genomic map of Bz181. The inner circle represent the reference sequence Bordetella pertussis Tohama I, following by \% G+C content, Bz181 genome and the location of virulence factors classes (colour labels, outer circle): adherence, endotoxin, motility, protease, regulation, secretion system, serum resistance and toxin. The Bz181 genome contigs were ordered according to B. pertussis Tohama I genome and the figure was performed using Blast Ring Image Generator (sourceforge.net/projects/brig).

comparative genomic analysis was conducted using the complete genomes of the B. pertussis strains Tohama I (accession BX640411) and CS (accession CP002695) as well as the resources generated by the classical Bordetella subspecies genome projects available at GenBank as of July, 2014. The Bz181 genome sequence was submitted to GenBank under accession SRS666189.

The sequences of genes encoding the pathogen-associated virulence factors were retrieved from the Bz181 genome and used in the $B$. pertussis multilocus sequence typing scheme (van Loo et al. 2002). The prn2-ptxS1Afim $3 \mathrm{~B}-p t x \mathrm{P} 3$ profile was obtained, which corresponded to that observed in the current strains causing pertussis worldwide (van Loo et al. 2002, Kallonen et al. 2011). The set of virulence factor-encoding genes that is under the control of the global BvgAS regulatory system (Aricó et al. 1989) was also identified in Bz181 (Figure).
Unfortunately, there is no genomic information available for the B. pertussis strain used in the current Brazilian vaccine. This information would provide insight concerning the scenario of transmission and the possible causes of pertussis in Brazil.

Sequencing of the Brazilian B. pertussis Bz181 genome revealed the presence of a putative, unique coding sequence (GenBank accession KM668553) that presented all of the conserved zinc-binding motifs that are part of the subclass B3 metallo- $\beta$-lactamase catalytic site and are crucial for enzymatic activity (Garau et al. 2004). Although identical sequences are present in B. pertussis Tohama I (GenBank accession BX640411; locus tag BP 230) and B. pertussis CS (accession CP002695; locus tag BPTP 299), they have not been assigned as a metallo- $\beta$ lactamase-like genes in these genomes. A search of the available resources from the classical Bordetella subspe- 
cies genome projects revealed the presence of this sequence in all $B$. pertussis genomes. However, in the $B$. parapertussis and $B$. bronchiseptica genomes, several different metallo- $\beta$-lactamase-like alleles were identified, including sequences with a nucleotide insertion resulting in a premature stop codon.

Classification of functional subsystems performed using RAST demonstrated the presence of putative genes associated with resistance in Bz181, including the multidrug resistance-related $\mathrm{CmeABC}$ efflux pump from the RND superfamily (membrane fusion protein CmeA, GenBank accession KM668555, inner membrane transporter CmeB, GenBank accession KM668556, outer membrane lipoprotein CmeC, GenBank accession KM668554). Although this efflux pump has never been reported in Bordetella, a search performed in GenBank revealed the presence of the cme operon in other Bordetella genomes. This efflux pump has been reported and characterised in Campylobacter and is involved in resistance to clinically important antibiotics used to treat infections caused by this organism, such as fluoroquinolones, erythromycin, tetracycline, rifampin, gentamycin and some $\beta$-lactams (Lin et al. 2002). Macrolides, especially erythromycin, have been the drug of choice for the treatment and postexposure prophylaxis of pertussis (Langley et al. 2004). Therefore, the presence of a putative CmeABC efflux pump in Bz181, which could evoke erythromycin resistance, is of concern and further experimental assays to determine its role and functionality must be performed. However, the mutations responsible for the emergence of fluoroquinolone resistance were not detected in the gyrA and parC sequences of Bz181.

The findings presented in this report provide relevant genomic information concerning the current epidemiological scenario of pertussis in Brazil, where the major pertussis immunisation policy is based on a whole-cell vaccine. The results of this work will contribute to a better understanding of $B$. pertussis evolution.

\section{ACKNOWLEDGEMENTS}

To PDTIS-Fiocruz and to Platform, for high-throughput sequencing of IOC-Fiocruz for use of their facilities.

\section{REFERENCES}

Aricó B, Miller JF, Roy C, Stibitz S, Monack D, Falkow S, Gross R, Rappuoli R 1989. Sequences required for expression of Bordetella pertussis virulence factors share homology with prokaryotic signal transduction proteins. Proc Natl Acad Sci USA 86: 6671-6675.

Bart MJ, Harris SR, Advani A, Arakawa Y, Bottero D, Bouchez V, Cassiday PK, Chiang CS, Dalby T, Fry NK, Gaillard ME, van Gent M, Guiso N, Hallander HO, Harvill ET, He Q, van der Heide HG, Heuvelman K, Hozbor DF, Kamachi K, Karataev GI, Lan R, Lutyłska A, Maharjan RP, Mertsola J, Miyamura T, Octavia S, Preston A, Quail MA, Sintchenko V, Stefanelli P, Tondella ML,
Tsang RS, Xu Y, Yao SM, Zhang S, Parkhill J, Mooi FR 2014. Global population structure and evolution of Bordetella pertussis and their relationship with vaccination. MBio 5: e01074.

Bart MJ, van Gent M, van der Heide HG, Boekhorst J, Hermans P, Parkhill J, Mooi FR 2010. Comparative genomics of prevaccination and modern Bordetella pertussis strains. BMC Genomics 11: 627.

Garau G, García-Sáez I, Bebrone C, Anne C, Mercuri P, Galleni M, Frère JM, Dideberg O 2004. Update of the standard numbering scheme for class B $\beta$-lactamases. Antimicrob Agents Chemother 48: 2347-2349.

Hartzell JD, Blaylock JM 2014. Whooping cough in 2014 and beyond: an update and review. Chest 146: 205-214.

Harvill ET, Goodfield LL, Ivanov Y, Meyer JA, Newth C, Cassiday P, Tondella ML, Liao P, Zimmerman J, Meert K, Wessel D, Berger J, Dean JM, Holubkov R, Burr J, Liu T, Brinkac L, Kim M, Losada L 2013. Genome sequences of 28 Bordetella pertussis US outbreak strains dating from 2010 to 2012. Genome Announc 1: e01075-13.

Kallonen T, Gröndahl-Yli-Hannuksela K, Elomaa A, Lutynska A, Fry NK, Mertsola J, He Q 2011. Differences in the genomic content of Bordetella pertussis isolates before and after introduction of pertussis vaccines in four European countries. Infect Genet Evol 11: 2034-2042.

Langley JM, Halperin SA, Boucher FD, Smith B, Pediatric Investigators Collaborative Network on Infections in Canada (PICNIC) 2004. Azithromycin is as effective as and better tolerated than erythromycin estolate for the treatment of pertussis. Pediatrics 114: e96-e101.

Lin J, Michel LO, Zhang Q 2002. CmeABC functions as a multidrug efflux system in Campylobacter jejuni. Antimicrob Agents Chemother 46: 2124-2131.

Mattoo S, Cherry JD 2005. Molecular pathogenesis, epidemiology and clinical manifestations of respiratory infections due to Bordetella pertussis and other Bordetella subspecies. Clin Microbiol Rev 18: 326-382.

Parkhill J, Sebaihia M, Preston A, Murphy LD, Thomson N, Harris DE, Holden MT, Churcher CM, Bentley SD, Mungall KL, CerdeñoTárraga AM, Temple L, James K, Harris B, Quail MA, Achtman M, Atkin R, Baker S, Basham D, Bason N, Cherevach I, Chillingworth T, Collins M, Cronin A, Davis P, Doggett J, Feltwell T, Goble A, Hamlin N, Hauser H, Holroyd S, Jagels K, Leather S, Moule S, Norberczak H, O’Neil S, Ormond D, Price C, Rabbinowitsch E, Rutter S, Sanders M, Saunders D, Seeger K, Sharp S, Simmonds M, Skelton J, Squares R, Squares S, Stevens K, Unwin L, Whitehead S, Barrell BG, Maskell DJ 2003. Comparative analysis of the genome sequences of Bordetella pertussis, Bordetella parapertussis and Bordetella bronchiseptica. Nat Genet 35: 32-40.

van der Zee A, Groenendijk H, Peeters M, Mooi FR 1996. The differentiation of Bordetella parapertussis and Bordetella bronchiseptica from humans and animals as determined by DNA polymorphism mediated by two different insertion sequence elements suggests their phylogenetic relationship. Int J Syst Bacteriol 46: 640-647.

van Loo IH, Heuvelman KJ, King AJ, Mooi FR 2002. Multilocus sequence typing of Bordetella pertussis based on surface protein genes. J Clin Microbiol 40: 1994-2001. 